\title{
An Indigenous Design for Management of Congenital Scoliosis - A Case Study
}

\section{Parthasarathi Swain ${ }^{1}$, Sonali Mohanty ${ }^{2}$, Bapina Kumar Rout ${ }^{3}$, Rojaleen Pradhan ${ }^{4}$}

\begin{abstract}
${ }^{1}$ MPO, Department of Prosthetics \& Orthotics, National Institute for Empowerment of Persons with Multiple Disabilities, Chennai, Tamil Nadu, India.

${ }^{2} \mathrm{BPO}$, is Affiliated with the Aditya Prosthetic \& Orthotic Center, Aditya Diagnostics \& Hospitals, Dibrugarh, Assam, India

${ }^{3} \mathrm{MPO}$, is Affiliated with the National Institute for Empowerment of Persons with Multiple Disabilities, Chennai, Tamil Nadu, India.

${ }^{4} \mathrm{MPO}$, is Affiliated with the Swami Vivekananda National Institute Rehabilitation Training \& Research, Olatapur, Odisha, India.
\end{abstract}

Corresponding Author: Parthasarathi Swain

\begin{abstract}
Introduction: Available orthotic brace options for congenital scoliosis are a few and are quite cumbersome to be tolerated by infants. Considering comfort, acceptance, and effectiveness, we have designed and developed a new spinal Orthosis, incorporating a flexible strap system. The purpose of this study was to investigate the effect of this novel flexible spinal Orthosis on scoliotic curve correction and progression.

Methods: A 1-year-old female infant diagnosed with congenital Scoliosis was fitted with the novel flexible spinal orthotic brace to control the curve progression and to maintain an upright posture. The anterior-posterior radiographs were analyzed for measuring the Cobb angle. The plumb line test was used to assess the postural improvements. Before and after tests were conducted with and without the Novel spinal brace with an intervention period of 2 months.

Discussion: The Cobb's angle was found to be $7^{\circ}$ less after the intervention period of 2 months. There was also a marked improvement in Body posture with a $6 \mathrm{~mm}$ plumbline shift towards the midline. This indigenous flexible spinal brace can be considered as a new, effective method for the treatment of Congenital Scoliosis and it also can be used for maintaining an aligned posture.
\end{abstract}

Key Indexing Terms: Congenital Scoliosis, Flexible spinal Brace, Cobb's angle.

\section{INTRODUCTION}

Congenital scoliosis is a lateral curvature of the spine that is due to the presence of vertebral anomalies that cause an imbalance in the longitudinal growth of the spine. ${ }^{1}$ A key feature of congenital scoliosis is the presence of one or more abnormally formed vertebrae. When these anomalies are identified, the curve should be classified as congenital, even if the deformity is not apparent until adolescence. Congenital scoliosis occurs very early in development; in the first six weeks of embryonic formation. ${ }^{2}$ Congenital scoliosis does not seem to run in families. Genetic studies to date have not yielded much evidence that this condition can be inherited. Although congenital scoliosis is often discovered during the infant or toddler period, in some children it is not diagnosed until their adolescent years. It is caused by inborn spinal deformity, which involves abnormalities in the development of vertebra i.e. absent or fused vertebra. ${ }^{3}$ The condition of scoliosis becomes more relevant in adolescent as the spine begins to 
grow more quickly, focusing more stress on the inborn abnormalities. Curvature in such cases must be monitored closely since the progress is more vigorous. ${ }^{4}$

Bracing plays a very limited role in the management of congenital scoliosis, especially as a primary form of treatment because these curves are typically inflexible. Moreover, the brace is also working in a high aesthetical sense. This case study aimed to quantify the amount of curve correction and to prevent further progression of the curve in congenital scoliosis, the postural improvements without sacrificing the biomechanical principles of normal spine and comfort of the patient.

\section{MATERIALS AND METHODS SUBJECT}

The child patient was a 1-year-old girl who has congenital scoliosis with spinal curvature of $34^{\circ}$. She was referred to the Department of Orthotics and Prosthetics in the Swami Vivekananda National Institute of Training and Research (SVNIRTAR), for the provision of a suitable spinal Orthosis. The patient gave a written informed consent form to participate in the study, and appropriate approval was also obtained from the Institutional Ethical committee. The child's cognitive functions were normal, and she was interactive. A detailed assessment was performed with demographic data, medical history, $\mathrm{x}$-ray evaluation, and Cobb angle, angulations in the sagittal and frontal plane, and functional outcome.

\section{FABRICATION PROCEDURE}

TABLE - 1. Result of cobb's angle and plumb line test

\begin{tabular}{|l|l|l|l|}
\hline $\begin{array}{l}\text { Outcome } \\
\text { measures }\end{array}$ & $\begin{array}{l}\text { Measurement } \\
\text { before the } \\
\text { intervention }\end{array}$ & $\begin{array}{l}\text { Measurement } \\
\text { just after the } \\
\text { intervention }\end{array}$ & $\begin{array}{l}\text { Measurement } \\
\text { after 2months } \\
\text { of the } \\
\text { intervention }\end{array}$ \\
\hline $\begin{array}{l}\text { Cobb's } \\
\text { angle } \\
\text { (degrees) }\end{array}$ & 34 & 29 & 27 \\
\hline $\begin{array}{l}\text { Plumb } \\
\text { line } \\
\text { test }(\mathrm{mm})\end{array}$ & 17 & 13 & 11 \\
\hline
\end{tabular}

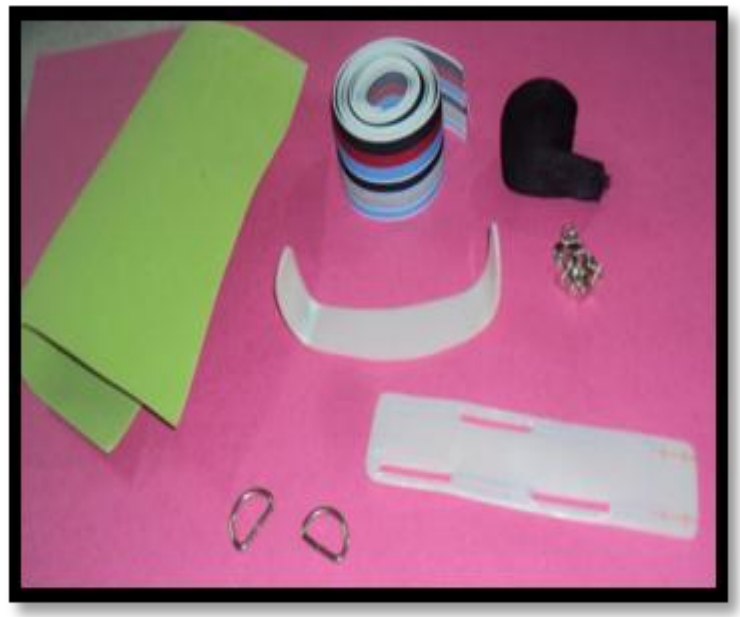

Fig 1. Components of Orthosis

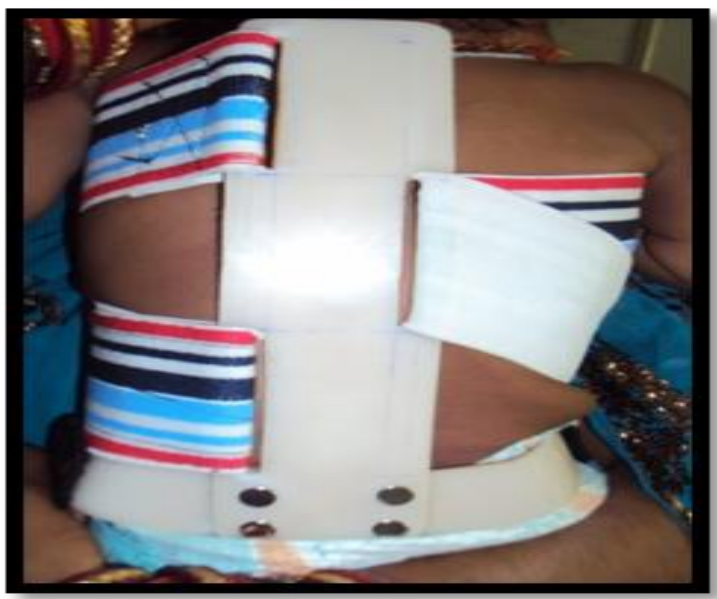

Fig 2. The flexible spinal brace with the subject

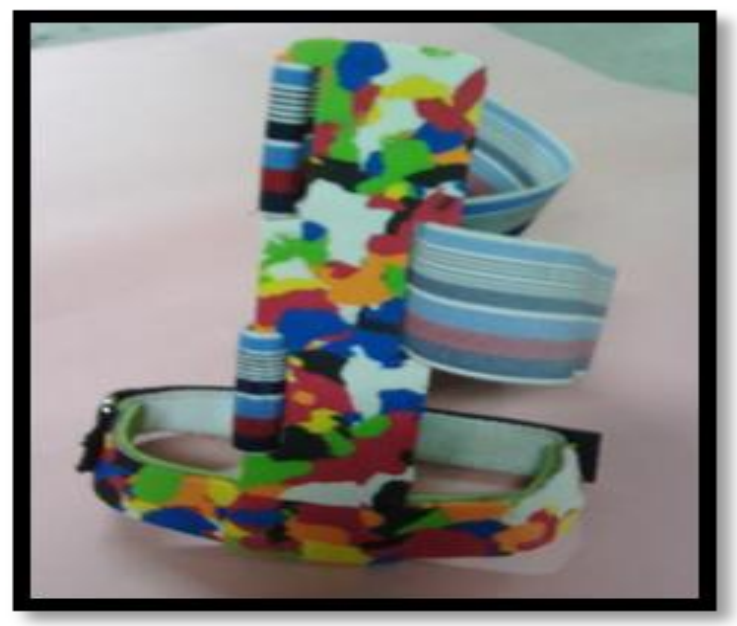

Fig 3. The back view of the spinal brace with the elastic bands providing a three-point force system to prevent the curve progression

This Orthosis was made up of three components, one pelvic section, one posterior upright, and one flexible strap. Both pelvic section and posterior upright gave support to the Orthosis as it was made up of polypropylene and a flexible strap 
encircle the torso and aligned the trunk. Pelvic Section acted as an anchoring point, made up of a $3 \mathrm{~mm}$ polypropylene sheet. It formed the foundation for the Orthosis in which the posterior upright was attached and anteriorly it encircled the torso.

Being made up of flexible polypropylene, the width and thickness of the Posterior upright were approximately $50 \mathrm{~mm}$ and $3 \mathrm{~mm}$ respectively. It was centrally placed over the paraspinal muscles and attached distally with the pelvic band through rivets.

Proximally it extended half-inch below to the seventh cervical vertebra and distally up to the coccyx. It was shaped according to the normal curvature of the spine. Three slots are made, equidistance to each other for the proper execution of force by an elastic strap. It also provided posteriorly directed force and helped to maintain the spine erect. One single elastic strap was used and applied in a spiral manner from the posterior-anterior-posterior direction. Likewise, it provided 3 point force system, one corrective force at the apex of curvature (convex side), and two opposing forces at the level of end vertebrae (concave side).

\section{DISCUSSION}

After two months of follow up the Cobb's angle had been reduced in this case and the aesthetical sense has improved due to trunk symmetry. Result of this case report was analyzed with the comparison of Pre and Post-Test Cobb's angle \& amount of apical vertebrae deviation from mid-line. Though there was a small degree change in the Cobb angle and progression of the curve. There was also improved posture with a shift in the plumb line $6 \mathrm{~mm}$ towards the midline with the use of a brace (Table $1)$.

As this Orthosis is based on the flexible brace concept so it minimizes the physical constraint and includes compliance. The flexible nature of the pelvic section of the Orthosis permits free movements of the trunk and engagement of the pelvis in the corrective movement. The pelvic band acts as an anchoring point and supports the action applied to the patient's trunk by elastic bands. The principle of "mechanical biofeedback therapy" is used to control scoliotic deformity by applying a dynamic harness.5 The harness provides dynamic control of the trunk, limits undesirable movements, and modifies the 3D postural geometry while preserving body movement and growth.

This study has also confirmed that the flexible brace treatment along with physiotherapy is an effective way to treat congenital scoliotic patients less than one year of age, which alters the natural history of the disease. As surgery is least indicated for the very small age group, so conservative treatment through spinal braces is the best option.

\section{CONCLUSION}

This study concludes that the flexible brace design is an effective way of brace treatment for congenital scoliosis in comparison to other designs. This is an innovative design that generally attends to the congenital scoliosis deformity of the spine. It is easier to prevent the progression of the small curve than to reverse the deformity in a large curve. It provides motion control and maintains a static posture of the spine by creating a 3-point force system. It increases spinal stability by applying external forces to substitute for defective muscle sections $\&$ thereby reduces pain. This brace is more successful than the previously existing design. Based on our present analysis, we observed better comfort and functional outcomes with the use of this new spinal brace design manufactured with this new technique. It should be emphasized that because of the uni sample size nature of this study, the validity of this intervention should be reconfirmed by a large sample size and randomised clinical trial.

\section{Acknowledgement: None}

Conflict of Interest: None 


\section{Source of Funding: None}

\section{REFERENCES}

1. Janicki JA, Alman B.. Scoliosis: review of diagnosis and treatment. Paediatr Child Health. 2007;12(9):771-776.

2. Arlet V, Odent T, Aebi M. Congenital scoliosis. Eur Spine J, 2003, 12: 456-463.

3. Burnei G, Gavriliu S, Vlad C, et al. Congenital scoliosis: an up-to-date. J Med Life. 2015;8:388-97.

4. Dunn J, Henrikson NB, Morrison CC, Nguyen M, Blasi PR, Lin JS. Screening for Adolescent Idiopathic Scoliosis: A Systematic Evidence Review for the U.S.
Preventive Services Task Force [Internet]. Rockville (MD): Agency for Healthcare Research and Quality (US); 2018 Jan. Report No.: 17-05230-EF-1. PMID: 29638297.

5. Giggins O.M., Persson U.M., Caulfield B. Biofeedback in rehabilitation. J. Neuroeng. Rehabil. 2013;10:60.

How to cite this article: Swain P, Mohanty S, Rout BK et.al. An indigenous design for management of congenital scoliosis - a case study. Int J Health Sci Res. 2021; 11(5):196199. DOI: https://doi.org/10.52403/ijhsr. 20210530 